\title{
A DESIGN INQUIRY INTO THE ROLE OF DESIGN PROCESS IN FOSTERING CREATIVE EXPLORATION OF IDEAS AND CONCEPTS: AN EXPLORATORY STUDY OF DESIGN PROJECTS OF ENGINEERING STUDENTS
}

\author{
Purba JOSHI and Sharmila SINHA \\ Indian Institute of Technology Bombay, India
}

\begin{abstract}
Creativity forms the base for innovation. Thus, facilitating thinking outside of one's expertise and allowing the scope of exploration to build new knowledge becomes the challenge. Creative thinking often results from transferring problem solutions from one industry or domain to another through fusion of knowledge that has not been connected before. In the idea generation process, external sources of information are tapped to exploit multiple resource domains facilitating divergent exploration. While developing solutions, one is only able to notice a limited section of the environment due to constraints of limited cognitive abilities and established thinking patterns [1].

To analyse this, an exploratory study was conducted with undergraduate engineering students and their application of the generative design process in coming up with design solutions for given design tasks. Results demonstrated that divergent \& convergent thinking stimulated opportunity identification and solution generation, thus improving the originality and diversity of ideas. Kulogun et al mentions, "To perceive, interpret and communicate the world and the facts differently is an important goal of design education and can only be obtained through creative thinking" [2].

This study aims to identify the generative stage of the creative process and help enable opportunities for creative ideation. The study suggests that design education in non-design disciplines can enhance creative exploration and facilitate innovative problem-solving solutions. The generative framework can further help define pedagogic interventions for teaching design process to large classes of nondesign students.
\end{abstract}

Keywords: Creative thinking, idea generation, design process, design teaching

\section{INTRODUCTION}

While developing solutions, designers are only able to notice a limited section of the environment due to their limited cognitive abilities and established thinking patterns [1]. Design fundamentally follows a step-by-step procedure in procuring and implementing knowledge. To understand the creative process involved in design it is necessary to analyse the design process as active steps towards design solutions that help enhance individual creativity.

To facilitate adequate idea exploration, inter-disciplinary knowledge can help in exploiting multiple domains to provide fertile and versatile grounds for enhancing creative exploration. Creative thinking often results from transferring problem solutions from one industry or domain to another through fusion of knowledge that has not been connected before. Creativity involves searching for new knowledge.

This paper presents an exploratory study, conducted with undergraduate engineering students who applied the generative design process in coming up with creative design solutions for a given task. The study aims to analyse each stage of the creative process and help redefine opportunities for creative representation to maximise creative idea and concept generation. The study suggests that design education in non-design disciplines can enhance exploration and facilitate innovative solutions. 


\subsection{Aim of the study}

This study explores the possibilities of facilitating innovative problem solutions through the implementation of the 7C's design process [3] in non-design disciplines by presenting two project cases. For triggering exploration and creative conceptual outcomes, a simplified yet extensive process can be beneficial. Another challenge during the study was to communicate and implement the design process in a class of 127 engineering students from different disciplines.

This study aims to build knowledge on the role of a comprehensible design process in fostering creativity in engineering students, thereby exploring the potential of the 7Cs design process as an integral part in the creative ideation and conceptualisation of products. Additionally, this can help develop a methodology for pedagogical interventions to enhance idea exploration and understanding of design process.

\subsection{Design process}

Researchers have tried to develop systems model to aid the creative idea generation. They have described and tried to formalise the design process by producing generic steps using the cognitive process.

\subsubsection{Design process and Creative concept generation}

Methods and strategies of creative problem solving try to initiate an operating system of innovation. Product design too focuses on similar processes. As the exploration of ideas is an important part of creativity, it is important to consider and nurture exploration in the design process [4]. Thus, a systematic generative design process can help in keeping mental inertia in abeyance and allow radical thinking.

Psychological aspects of thinking patterns underline a dominant part of creativity and mental stimulation to generate new ideas. The overall creative problem-solving process has been explained in four steps: Preparation, Incubation, Illumination, and Verification [5]. This has formed the basis of all consequent creative thinking / problem solving endeavours. Over time, this has been further detailed into five steps: Problem Formulation, Preparation, Idea Generation, Idea Evaluation and Idea Selection [6]. Creative exploration is of highest importance in the design scenario, so as to compete by generating new opportunities. New and creative ideas emerge from a conscious effort to balance analysis and imagination [7] and by expanding and varying the search space of alternatives for design possibilities to inspire creativity [8]. Still, certain comprehendible challenges seem to arise in its application by non-design students. Thus, a more generative design process that is systematic, organised and inter-connected for effective and sustained functioning, will lead to a continuous generation of new ideas and creative exploration.

\subsubsection{The 7C's Design Process}

As mentioned earlier, the 7Cs design process for innovation is a structured step-by-step framework that elucidates the developmental journey of a design to its creative output. The seven steps are [3]:

Cause - the concern to solve a problem by identifying needs and design opportunity.

Context - understanding the user, the scenario and parallel products in the market.

Comprehension - arriving at insights by analysing all available data gathered and drawing inferences. Check - a clear plan of action to set the design brief based on the design insights. This defines criterion for ideation and concept evaluation.

Conception - creating multiple ideas and combining them to generate concepts for further development.

Crafting - detailing and developing final concept. Depending on the complexity and requirement of the project a small scaled mock up, full-scale model, working rig or CAD models are created for visual representation of the idea.

Connection - connecting the solution provided with the user need. Getting user feedback on the proposed design to predict its demand and success in the market.

The operational process shown in figure. 1 helps to describe the design activity as a whole and depicts the steps for a holistic problem-solving application. 

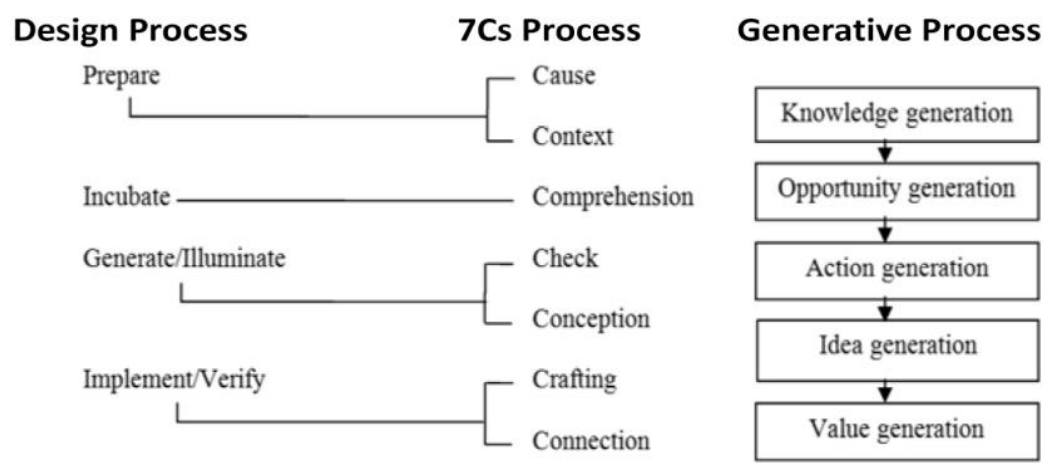

Figure 1. Evolution of 7C's generative framework [3]

\section{METHOD}

The design project cases were part of a graded assignment within a university course - 'Introduction to Design' for undergraduate engineering students from different disciplines who were in their second and third year of studies. During the course, they were taught the 7Cs design process through lectures and design case studies. The implementation culminated in a conceptual class project. The progressive design ideas while applying the generative framework, presentation, reports and documents were used for analysis.

An explorative approach of study is taken towards two selected design project cases to further the understanding of the implications of the 7Cs generative framework's workings and its influencing factors on design exploration. To generate contextual knowledge of the given cause, study of users, parallel situations as well as lateral situations were carried out to identify constraints, scope and insights to be able to generate opportunity and ideas using analogy triggers.

The process is further elaborated in figure. 2 .

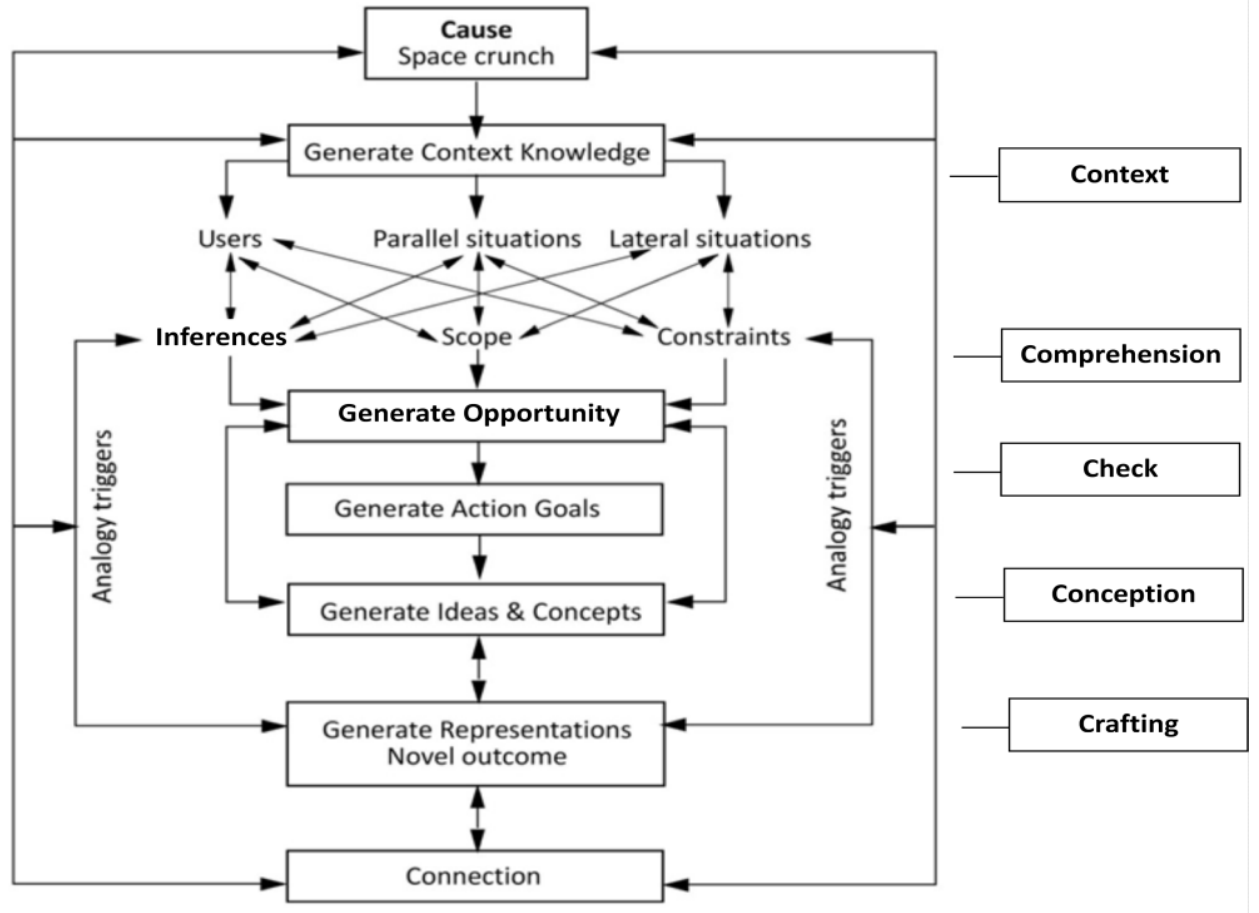

Figure 2. The Operational Flow of 7C's generative framework [9]

The framework helped focus on generating design opportunity, insights and generate solutions within two space crunch scenarios. Being an extensive research and implementation process, the tasks were performed in groups over the span of the entire semester. The projects were broken down in 7 stages arising out of 7C's. Each stage was discussed in the class and each group was required to carry out tasks in order to complete the stage and make a short presentation after every stage. They were instructed to submit a report at the end of the semester explaining their design journey as well as make 
a short presentation to receive their final grades and fulfil the course credits. A brief description of the data produced are presented below:

\section{Project 1}

Inadequate space in hostel rooms for study table, resulting in postural issues

User study with students and hostel management. Study of parallel products. Find how else space optimization is carried out.

Inferences from user study and other knowledge sources to identify constraints and scope based on no: of occupants visavi room size, and affordability

i)Space optimization, foldable and compact ii) Ergonomic and comfortable. iii) Robust. iv) Easy to fit within available space. v) Multi purpose builtin useful features

Thoughts and insperation. Foldable/compact, Sliding/Layers, Suspended, Expandable/adjustable, Multi use

Analogy: Cloud, bullet, tree, dew on leaf, cake, stapler, swan, waves, bud, tripod, antenna, flip phone, hanging projector
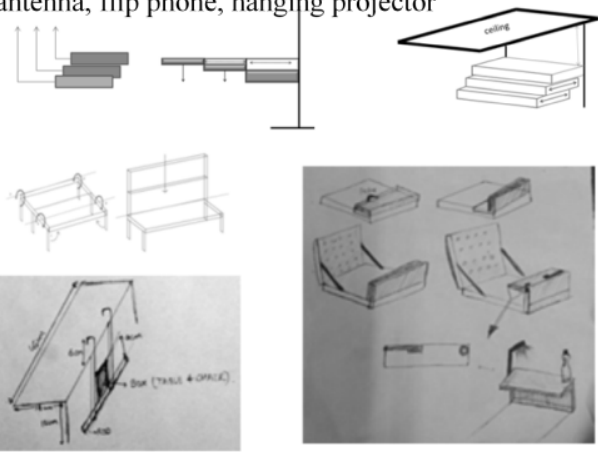

Detailed mechanical drawing
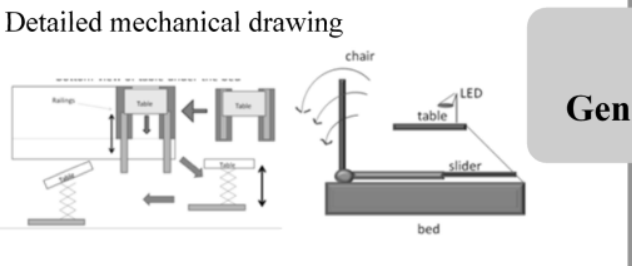

Matching design outcome to need innovatively. Detailed function depicting drawing to explain usibility

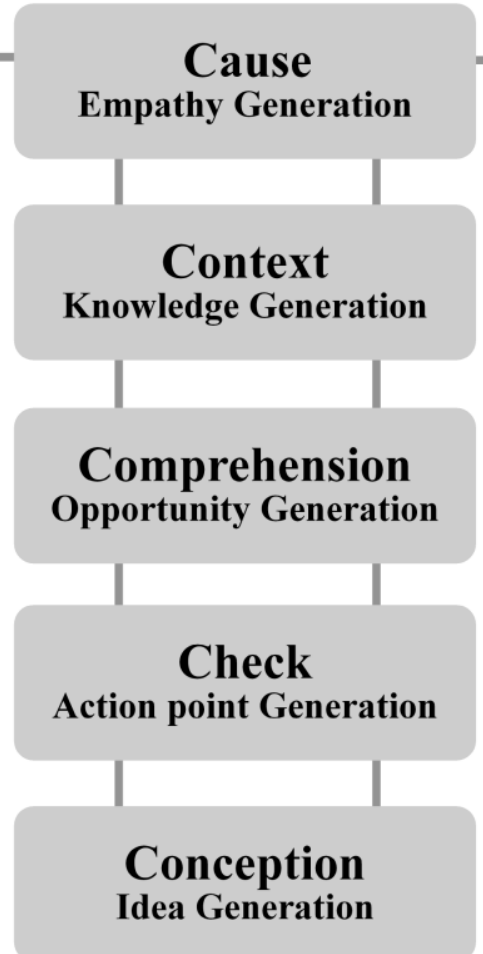

Space crunch in hostel mess during peak hours

User study with students and mess maintenance staff. Study of parallel products. Find how else space optimization is carried out.

Inferences from user study and other knowledge sources to identify constraints and scope

i)Space optimization and maneuveability ii) Stackability to facilitate cleaningmess area and space generation. iii) Economicalcost effective. iv) Style and comfort v) Multi usage features- storage space for students to keep belongings

Thoughts and inspiration.

Foldable/compact/step, Strong and light weight, Hang/keep, Flexible and

Secure/lock, beauty

Analogy: Peacock, hot air balloon,

Kangaroo, ladder, lock, spectrum, ring,

cloud, hanger

Water proof. Compact, detachable storage,

Expandable storage, multi compartment
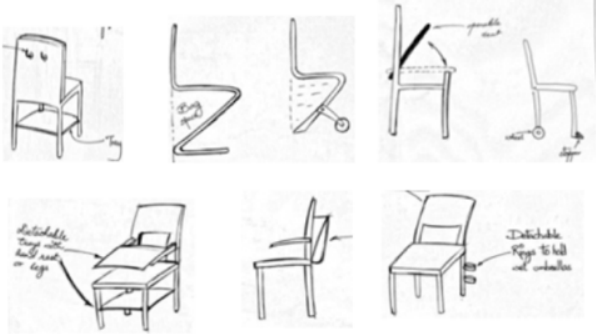

CAD Model

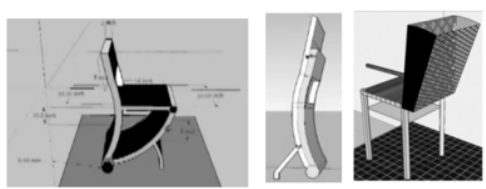

Matching design outcome to need innovatively. Simulate product form and function through $\mathrm{CAD}$ model

Figure 3. The 2 projects implementation of 7C's design process [3]

\section{DATA ANALYSIS}

The study draws inferences from the project cases in order to establish the 7Cs generative framework in supporting creative ideation and the development of product solutions. The ideas and design thoughts that were generated were analysed and rated on their originality, flexibility and relevance [10]. The rate scores are depicted in the table 1. 
Table 1. Creativity ratings and idea output using the 7Cs Generative Framework

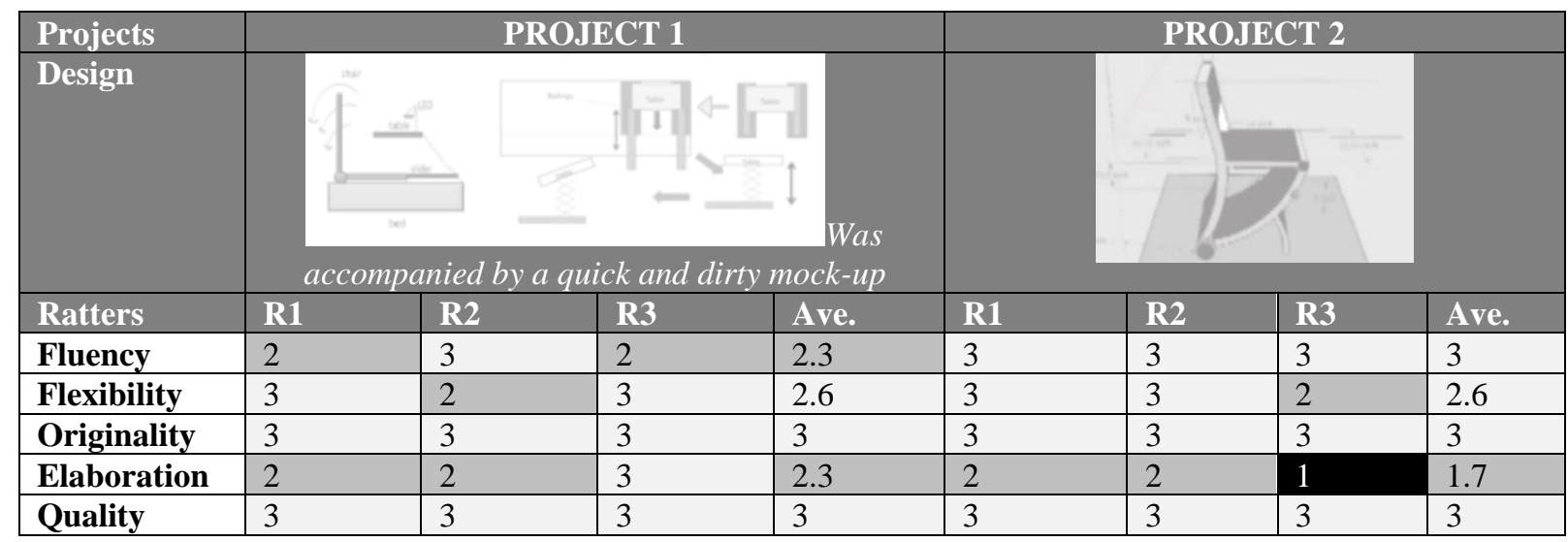

\section{3-2.4=High $\square$ 2.3-1.7=Moderate $\square$ Below1.6=Low}

Due to the generative process, at every level diverse exploration were carried out. The opportunity generation triggered primary action thought links that helped in generation of multiple sub-idea links. The results showed high fluency, flexibility, originality and usefulness, of the ideation outcome thus giving the mark of creative ideas produced by the 7Cs generative framework by non-design students. This exploratory approach of inquiry was used to get an understanding of the challenges and advantages of the 7Cs generative framework for product design \& engineering design implementation.

\section{FINDINGS AND DISCUSSIONS}

The findings of the inquiry show that generation of opportunity, creative idea and conceptual solutions exploration, has favourable effect on the design outcomes and its innovativeness by following the 7Cs process [3] within a Generative Framework [9]. The methodology helped to integrate associative thinking processes through analogy triggering at various stages to generate creative outputs. The generative phases of the framework helped manipulation of differing contexts to generate creative conceptual thought. The analogical association from a mix of natural as well as man-made objects steered abstract and lateral exploration by the fitting of attributes from source to domain [11]. The generative process helped the students to carry out exploratory thinking at every stage and develop novel design solutions. Thus, the idea output and the design outcomes indicated that the explicit framework at multiple stages facilitated diverse exploration for non-designers, this could enhance and bring benefit to projects at hand.

\section{CONCLUSIONS}

Studies on the creative thought process have highlighted certain characteristics of individuals in aiding creative processes: receptive attitudes, concern and involvement with problem, motivation, curiosity that helps to analyse and trigger alternate viewpoints. Thus, being sensitive to a situation, questioning its flaws, discovering what is missing and, moreover, to finally coming up with novel solutions that are applicable to desired users is fundamental to creativity in practice. Yet most often conventional intelligence takes centre stage and inhibits lateral exploration, thus compromising on the ideation output. Taking these nuances of the fundamentals, the 7Cs generative design framework was formulated and implemented to fulfil the design requirements of creative productivity in a more holistic way. It is a conscious process to break conventional thinking patterns with the use of analogy triggers to build diverse associations as articulated in the project examples.

The 7Cs generative framework combines both divergent and convergent thinking for generative purpose has helped facilitate exploration and solution implementation through diverse associations at multiple stages of design creation. The generative process when coupled with analogical association, it is able to move exploration to multiple diverse directions and deliver creative design ideas. The training in 7Cs generative framework can be rendered useful for exploratory product design projects. 


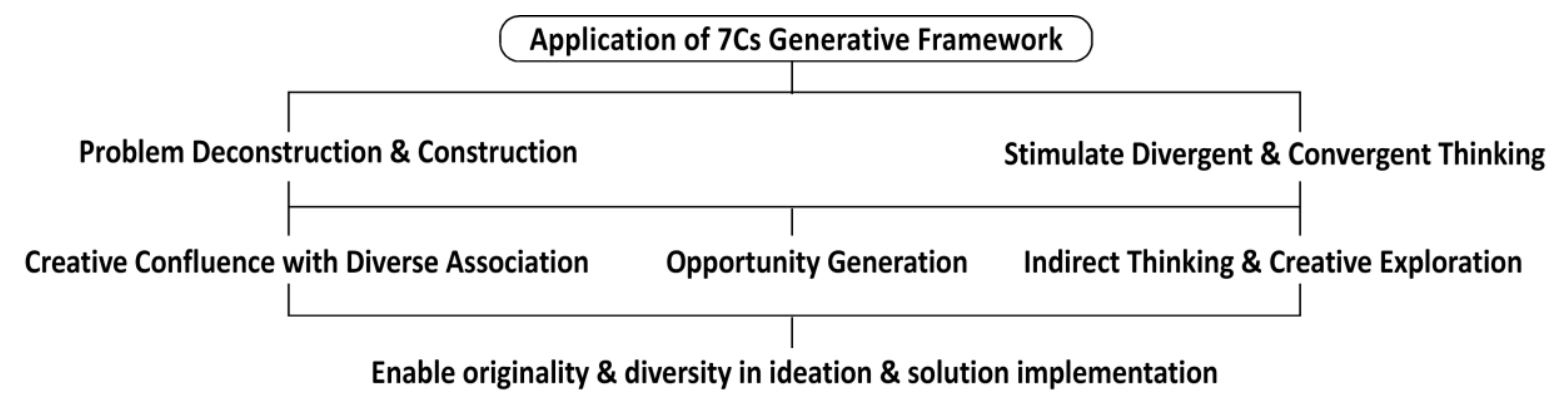

Figure 4: Outcome of 7Cs generative framework

Education today in non- design disciplines, like engineering and management, can enhance the creative productivity of both the students and their creative output through integrated pedagogic interventions. Implementation of creative generation and exploration of thought at every level of design development, can add value to the creative output, by enhancing the creative productivity and contributing positively to building a creative economy. Further work will be needed to formulate pedagogical instruments of implementing the 7Cs generative framework in non-design disciplines.

\section{ACKNOWLEDGMENT}

We are grateful to the 'Introduction to Design' course students of Indian Institute of Technology Bombay, India, for participating with full enthusiasm and rigor to complete and share their projects.

\section{REFERENCES}

[1] Simon H. Bounded rationality. Eatwell, J. e. a. (Eds), 1996 (The New Palgrave, London: Macmillan).

[2] Kuloglu N. and Asasoglu A.O. Indirect expression as an approach to improving creativity in design education. Procedia Social and Behavioural Sciences, 9, 2010, pp.1674-1686.

[3] Chakravarthy B.K. and Krishnamoorthi J. Innovation by Design: Lessons from Post Box Design and Development. Springer Science \& Business Media, 2013.

[4] Finke R.A., Ward T.B. and Smith S.M. Creative Cognition: Theory, Research, and Applications. MIT Press, 1992, Cambridge, MA.

[5] Wallas G. Stages in the Creative Process. The Creativity Questioned. Albert Rothenberg, Carl R. Hausman, Duke University Press, 1926, pp. 69-73.

[6] Amabile T. The Social Psychology of Creativity, Creativity in Context, 1983.

[7] Treffinger D.J. and Reis S.M. Creativity and Giftedness. Sage Publications, South Asia, 2004.

[8] Gero J.S. Creativity and Design. Artificial Intelligence and Creativity ed. T. Dartnall, 1994, pp 259-267.

[9] Sinha S., Joshi P. and Chakravarthy B.K. Fostering Creative Ideation: A Case of Idea Stimulation with Analogy. International Journal of Research and Analytical Reviews special issue, 2019, pp 387-392.

[10] Torrance E.P. The Torrance Tests of Creative Thinking Norms-Technical Manual Figural (Streamlined) Forms A and B. Bensenville, IL: Scholastic Testing Service 2008

[11] Sinha S. and Chakravarthy B.K. A design inquiry into the role of analogy in form exploration: an exploratory study. Proceedings of ICoRD13, Springer, 2013. 